Journal of Engineering and Applied Sciences 14 (1): 224-232, 2019

ISSN: $1816-949 \mathrm{X}$

(C) Medwell Journals, 2019

\title{
Authentication on Smartphones based Iris Recognition
}

\author{
Rana Jassim Mohammed, Taha Mohammed Hasan and Naji Mutar Sahib \\ Department of Computer Sciences, College of Science, University of Diyala, Diyala, Baqubah, Iraq
}

\begin{abstract}
The prevalent utilization of smartphones with the connectivity of the internet has led to sensitive data storage and transmission. This has increased the necessity to implement reliable user authentication on smartphones for preventing an attacker from reaching such data. The biometrics indicates the science of individual's recognition depending on their behavioral and biological traits. In order to perform reliable verification in smartphones, we briefly discuss the suitability of using the iris texture for biometric recognition in smartphones. In this study, we propose a new and robust iris segmentation method based on the circular distribution of angles to localize the iris boundary which applied on an eye noisy image passed through the pre-processing operation. For feature extraction, Zernike moments is used. After feature extraction, matching is performed by KNN. The proposed system has been experiment on the MICHE-I (Mobile iris Challenge Evaluation) iris dataset (Samsung Galaxy S4 (SG4) iris image database), featuring subjects captured indoor and outdoor under controlled and uncontrolled conditions by means of built-in cameras aboard three among the most diffused smartphones/tablets on the market. The evaluation of the obtained results show that the developed system can provide successfully iris recognition on the tested noisy imagesunder difficult environments compared to previous techniques and we have achieved $80 \%$ Av. accuracy rate with SG4.
\end{abstract}

Key words: Smartphones, iris segmentation, circular distribution of angles, MICHE-I dataset, Zernike moments, KNN

\section{INTRODUCTION}

The mobile phones that are able to be used as small computers and connect to the internet are referred to as "smartphones". Although, about 20 years ago the first smartphone-IBM Simon was presented, yet the world has witnessed a massive evolution of smartphones occurred when the first iPhone was introduced in 2007. Smartphones that are currently carried by humans are not only computers, databases, phones, cameras, locators, infinite jukeboxes and have the information in the world at human's fingertips but also personal companion which is a part of their everyday life. The role of smartphones as guardians, helpers and companions is speculated grow much bigger (Thavalengal, 2016).

The number of mobile phone users worldwide is expected to pass the 5 billion mark by 2019 and 2018, the number of tablet computer users is projected to reach 1.43 billion. This proliferation of smartphones and tablets raises concerns about the security and privacy of data stored on mobile devices if they are lost, stolen or hacked. An attacker with physical access to a mobile device can potentially steal a user's banking information, read his/her emails, look at his/her private photos and perform other criminal actions. The scale of the problem is vast; according to consumer reports.org, 2.1 million mobile phones were stolen and 3.1 million phones were lost in 2013 in the United States alone (Mitra and Gofman, 2016). For the purpose of balancing the user convenience with the necessary security by these smartphones, a mechanism is used to authenticate the users regularly with something with the users are or what users doing. This attribute should be unique for each user and not to be easy to mimic or stolen. It's possible to use biometrics for this purpose (Thavalengal, 2016). The science of person's recognition based on their behavioral or physical attributes is referred to biometrics. Some behavioral characteristics that are used to identify users are keystroke dynamics, signatures and voice patterns, while physical characteristics include retinal patterns, palm prints, hand geometry, fingerprints and iris patterns (Prabhakar et al., 2003). Among the different types of physical characteristics that are available, the iris is the most common and highly adopted biometric attributes because of its universality, accuracy and persistence. Recognition of iris is the method of identifying users based on their iris pattern (Jillela and Ross, 2015).

In this study, a comprehensive proposal for iris-based person authentication on smartphones is presented. It covers all the stages required to perform this

Corresponding Author: Rana Jassim Mohammed, Department of Computer Sciences, College of Science, University of Diyala, Diyala, Baqubah, Iraq 
task including eye acquisition, iris segmentation, features extraction and comparison to gallery images. In segmentation, stage proposes a new and robust iris segmentation method relies on the circular distribution of angles as a basis to localize the iris boundary in noisy images. For feature extraction Zernike moments is used, after feature extraction, matching is performed by (KNN), an extensive experimentation has been conducted implementing the proposed method on the publicly available smartphone iris dataset from BIPLabl on three among the most diffused mobile devices available on the market, Samsung's S4, Apple's iPhone 5 and Samsung's Galaxy Tab 2 which are generally considered good examples.

Literature review: Several methods have been proposed for iris segmentation in smartphones over the last few years. Raja et al. (2015) proposed a scheme to adapt to the visual iris based on the smartphone to achieve robust segmentation. The proposal shows that there is accuracy in $85 \%$ improved segmentation with OSIRIS V 4.1 . It is also used to extract a feature based on deep sparse filtering to obtain robust features for unrestricted iris images. Two different types of smartphones iPhone5S and Nokia Lumia 1020 were used to obtain a composite iris image database (VSSIRIS). The database (VSSIRIS) was obtained at $1.62 \%$ in EER andan average gain of around $2 \%$ in EER is obtained on the public database Compared to known modern schemes. Barra et al. (2015) presents a comprehensive method of iris authentication on mobile phones based on a spatial histogram. The proposed recognition algorithm performed well on reference iris datasets such as UPOL and UBIRIS while generally delivering worse results in the MICHE-I dataset which proved to be very difficult. Raja et al. (2017) proposed multi-patch deep features using deep sparse filters to obtain robust features for reliable iris recognition, a scheme has been proposed which has a high verification rat $(\mathrm{GMR}>95 \%)$ with low Equal Error Rate $(\mathrm{EER}<2 \%)$ through a series of intensive experiments on the MICHE-I iris dataset. In addition, simple distance measurements have been used to outperform the latest technologies to ensure that the proposed feature is robust. Additionally, the scheme is tested on the MПCHE-II challenge evaluation dataset where the results are promising with GMR $=100 \%$ on limited sub-corpus of iPhone data. Thavalengal (2016) proposed practical iris recognition solution for user authentication in next-generation smartphones, observed that using the existing smartphones 'as is' for iris recognition will not be an ideal solution in terms of user convenience, recognition performance and interoperability with the existing systems the experiments noted that these images suffer severely from the ambient noise, high-performing iris recognition algorithm-OSIRIS 4.1 is used in the experiments, using CASIA and MMU databases consist of NIR iris images. The technique is computationally light and does not require any additional hardware or significant change in the acquisition workflow while providing a mean error rate of $0 \%$ in the tested scenarios.

Based on three characteristics that describe different aspects of the iris which are the color, texture and characteristics of the clusters (color spots) that characterize the iris, a new and simple technique was introduced by Galdi and Dugelay (2017) to identify the iris. The performance was assessed on a subset of $\mathrm{MICHE}$ DB through images taken by two smartphones, Apple I Phone 5 (IP5) and Samsung Galaxy S4 (GS4). Additional experiments were performed by dividing the test group into two groups, one containing only IP5 images and one containing images obtained from GS4. The performance achieved in the subset of the images captured by the IP 5 device is very good with AUC of 0.98 . In fact, the low performance on GS4 images needs further investigation, possibly due to the fact that these images are more challenging because of the characteristics of the device or because of the different image resolution.A fast algorithm was proposed by Kim et al. (2016) for eye detection. As a result, away from the iris camera for the size of a $7 \times 13.7$ $\mathrm{cm}$ phone, two $850 \mathrm{~nm}$ LEDs were selected for iris illumination at $1.1 \mathrm{~cm}$. In the performance, the eye detection rate with the speed of $17.64 \mathrm{msec}$ on a mobile phone was $99.4 \%$ with recognition accuracy up to $1 \%$ EER (Equal Error Rate).

\section{MATERIALS AND METHODS}

The proposed system: The main goal of the proposed system is to develop a better-suggested system for iris authentication in smartphones using various operations as it is shown in Fig. 1.

Generally, the proposed system has some basic stages to perform all relevant recognition and verification tasks. The general structure of the typical iris authentication and verification system has five main stages. The first stage is the image acquisition and preprocessing stage, the second stage is the iris segmentation while the third and the fourth stages are the iris normalization and feature extraction and the last stage is the matching stage.

Image acquisition stage: Eye image acquisition is the first stage in the system. This stage depends on how much clarity and purity of the captured image are 


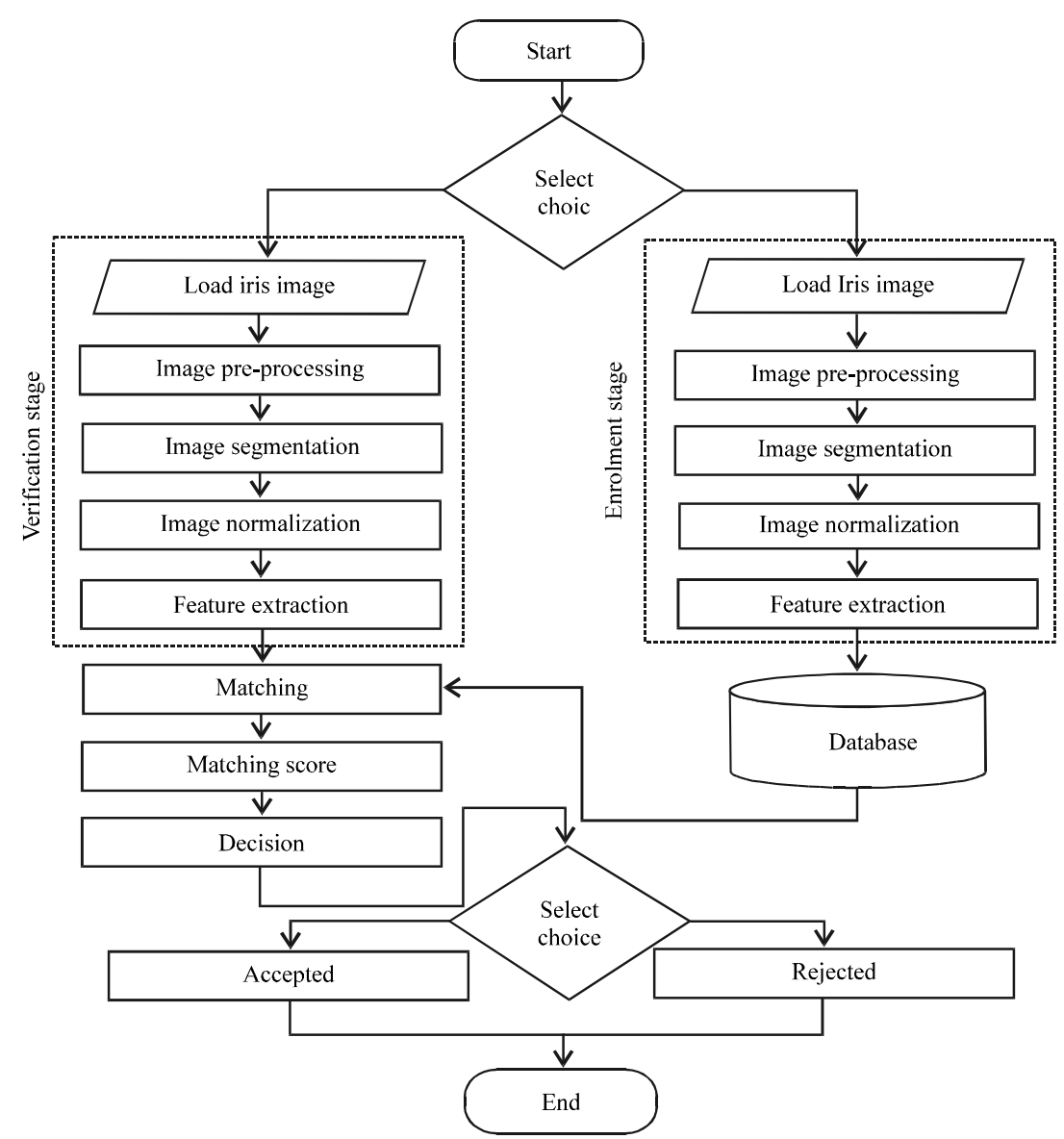

Fig. 1: The proposed system flowchart

provided. A specific class of iris images dataset is provided from the data set (MICHE)-I Mobile iris Challenge Evaluation, MICHE database contains photos captured indoor and outdoor with three different mobile devices; Samsung Galaxy S4 (hereinafter GS4), iPhone 5 (hereinafter iP5) and (Samsung Galaxy Tab 2), both the front and the rear cameras of this device is used. As we performed iris recognition, among the three devices, we selected the highest resolution cameras: GS4.

Image pre-processing stage: The pre-processing stage is necessary to prepare the iris image for further processing. Here, the image preprocessing consists of five steps: Find eye region, enhance eye image, convert the color image to the grayscale image, apply circler histogram to find the initial center of the iris image and finally, apply the global threshold to convert grayscale image to binary image. Figure 2 illustrates the preprocessing stage.

The first task of the pre-processing stage is to locate the eye region. To achieve this, the Haar cascade library is used as eye object detector one of the tools of open CV is implemented using $\mathrm{CH}$ language as shown in Fig. 3.

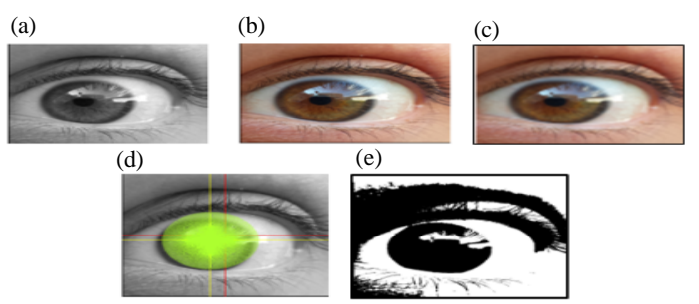

Fig. 2: Pre-processing stage: a) Eye region; b) Image contrast; c) Grayscale image; d) Apply circular histogram and e) Apply global threshold

(a)

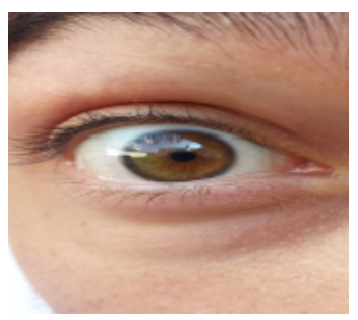

(b)

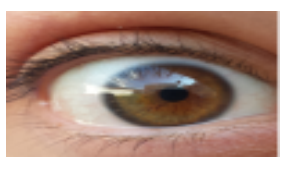

Fig. 3: Example of find eye region results using Galaxy S4; a) Original image and b) Find eye region 

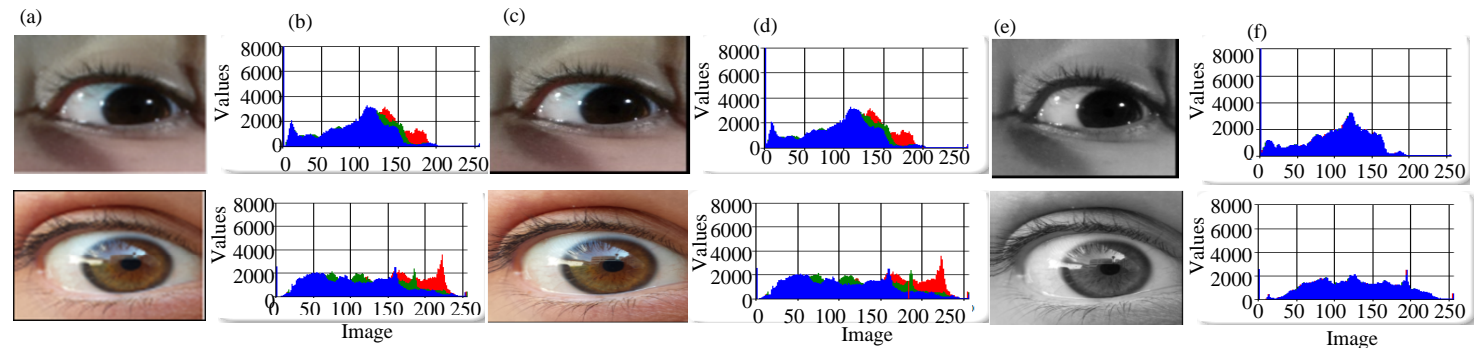

Fig. 4: Examples of contrast stretching; a) Original image; b) Histogram of original image; c) Image contrast; d) Histogram of image contrast; e) Grayscale image and f) Histogram of the grayscale image
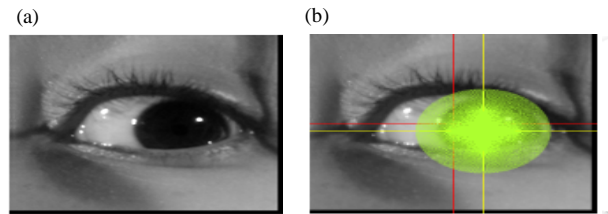

(c)
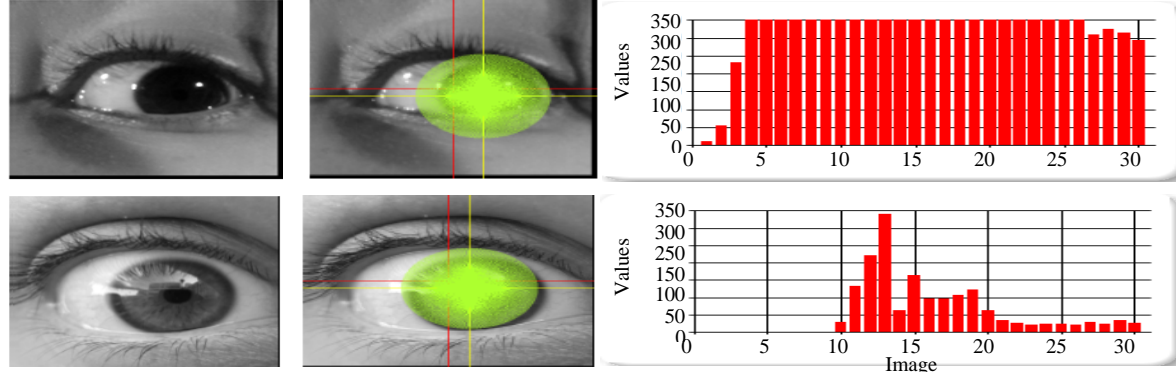

Fig. 5: An example of circular histogram; a) Grayscale image; b) Circular histogram and c) Histogram graphs in arranging (0-30)

The low contrast between pupil and iris is a noise factor that causes degradation in segmentation accuracy. To overcome this issue, enhancing the contrast is required before proceeding further to the iris segmentation process. In this work performing image contrast stretching using parameter valuebetween the range $[0.0-3.0], 0.9$ is the best coefficient that can be used for most images according to experience and make eye image region more distinguishable as shown in Fig. $4 \mathrm{c}, \mathrm{d}$. After that, converting the color image to a grayscale image by using a sophisticated version of the average method is the luminosity method. Figure $4 \mathrm{e}, \mathrm{f}$ illustratesthis conversation.

The fourth stage to find the initial center of iris image using the circular histogram. The histogram of an image is computed in the range $(0-255)$, here, the histogram is computed in a range (0-30) colors depending on the center of the original image based on Eq. 1 and 2:

$$
\begin{aligned}
& x c=\text { Height of image } / 2 \\
& y c=\text { Width of image } / 2
\end{aligned}
$$

With a default radius of 120 and angle from (0-360), we use the circle equation to draw a circle and draw the histogram of this space. The lowest occurrences number which not equal to zero in arrange (0-30) is taken to find the initial center of the iris regionas shown in Fig. 5.
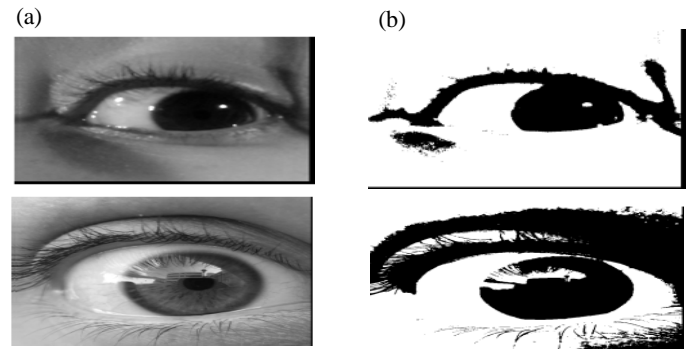

Fig. 6: Examples of Image conversation; a) Grayscale image and b) global threshold with the best threshold

The last stage in a pre-processing, apply global threshold creates binary images from grayscalelevel ones by turning all pixels below some threshold to zero and all pixels about that threshold to one. In our proposed system, the proper value of the Threshold (T) is estimated to be (127) as shown in Fig. 6.

Iris segmentation stage: It is the most important stage, since, all the sub stages based on the accuracy of this stage. This stage is applied for localizing and extracting the iris area from the image. The iris has a ring shape, could be roughly modeled as an annulus. To make the process simple and accurate, there are two steps required to segment iris region. Firstly, predicate 


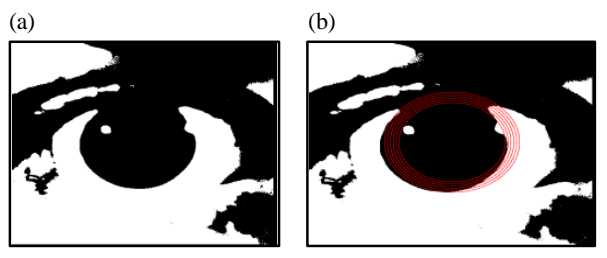

Fig. 7: An example of Predicate iris boundary; a) Binary image and b) Predicate iris outer boundary

iris outer boundary, secondly, a circular distribution of angles is applied to the segmented iris image.

Step 1; Predicate iris outer boundary: For predicting the iris boundary after getting: the approximate location of the iris center (xc, yc) which is computed from circular histogram as the initial center and initial radius (120 to0) with angle from $(0-360)$, we use the circle equation to draw a circle. This step is repeated till reaching the case that a significant ratio of circle pixels is black is equal to $100 \%$, new radius is calculated. The objective of predicate iris boundary algorithm is to asses more accurately the circle parameters that fit the collected of iri's segment as shown in Fig. 7.

Step 2; Circular distribution of angles: Because the iris was identified as having the largest black area (depending on the number of pixels) in the binary image. Therefore, the proposed system makes a scan for allocating the largest black segment in the binary image. After drawing the red circles and predicate iris outer boundary in the previous step. In this step, firstly, removing the existing specular spot reflections within the specified area; Secondly, the directions of each angles $(0,45,90, \ldots, 360)$ are determined to find the actual radius and center $(\mathrm{xc}, \mathrm{yc})$ of each iris as each angle consists of several points each point is considered the center and depending on the radius specified previously we draw a circle for each point to calculate the proportion of black and white after you take the largest percentage of blackness of the pixel density achieved by that angle and then calculate the radius and center for that angle to segmented the iris. This is the principle of circular distribution of angles. Figure 8 and 9 illustrate the circular distribution of the angles.

Table 1 and 2 describe the information of each angle and choice the angle that find the best center and radius of iris. Hence, localizing the iris by drawing a perfect geometry that fits the boundaries as shown in Fig. 10.

Iris normalization stage: After iris segmentation stage, the normalization stage uses circular distribution

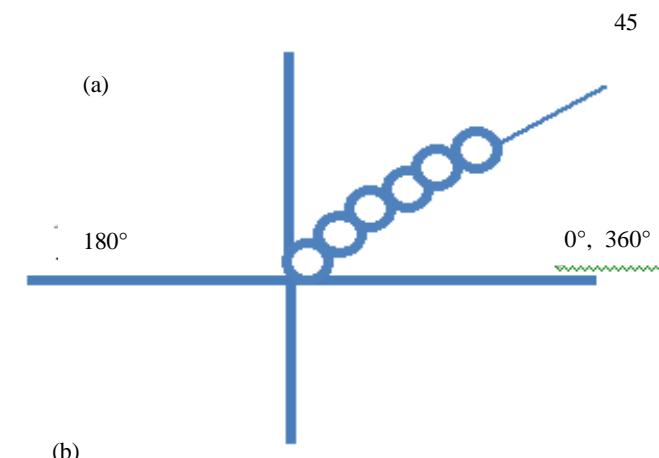

(b)

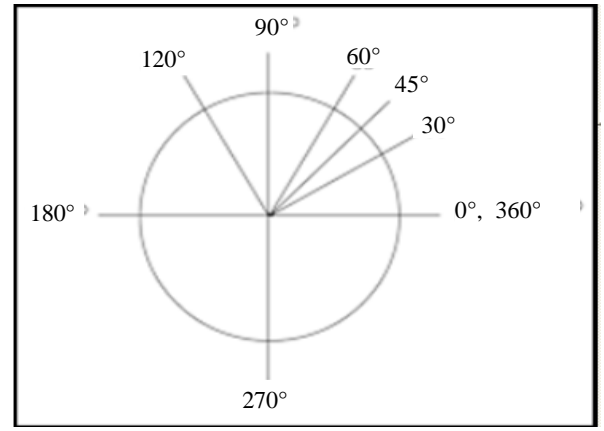

Fig. 8: Circular distribution of angles; a) Calculate circles in each angle and b) Directions of the angles

\begin{tabular}{llllllllllll} 
Table 1: Describe the information of each angle \\
\hline Center & 0 & 45 & 90 & 135 & 180 & 225 & 270 & 315 & 360 & 405 \\
\hline 0 & 0 & 0 & 0 & 0 & 0 & 0 & 0 & 0 & 0 & 0 \\
0 & 0 & 0 & 0 & 0 & 0 & 0 & 0 & 0 & 0 & 0 \\
0 & 0 & 0 & 0 & 0 & 0 & 0 & 0 & 0 & 0 & 0 \\
0 & 0 & 0 & 0 & 0 & 0 & 0 & 0 & 0 & 0 & 0 \\
0 & 0 & 0 & 0 & 0 & 0 & 0 & 0 & 0 & 0 & 0 \\
0 & 0 & 0 & 0 & 0 & 0 & 0 & 0 & 0 & 0 & 0 \\
0 & 0 & 0 & 0 & 0 & 0 & 0 & 0 & 0 & 0 & 0 \\
0 & 0 & 0 & 0 & 0 & 0 & 0 & 0 & 0 & 0 & 0 \\
0 & 0 & 0 & 0 & 0 & 0 & 0 & 0 & 0 & 0 & 0 \\
0 & 0 & 0 & 0 & 0 & 0 & 0 & 0 & 0 & 0 & 0 \\
0 & 96 & 97 & 73 & 56 & 64 & 75 & 71 & 62 & 96 & 0 \\
0 & 0 & 0 & 0 & 0 & 0 & 0 & 0 & 0 & 0 & 0 \\
0 & 0 & 0 & 0 & 0 & 0 & 0 & 0 & 0 & 0 & 0 \\
0 & 0 & 0 & 0 & 0 & 0 & 0 & 0 & 0 & 0 & 0 \\
0 & 0 & 0 & 0 & 0 & 0 & 0 & 0 & 0 & 0 & 0 \\
0 & 0 & 0 & 0 & 0 & 0 & 0 & 0 & 0 & 0 & 0 \\
0 & 0 & 0 & 0 & 0 & 0 & 0 & 0 & 0 & 0 & 0 \\
0 & 0 & 0 & 0 & 0 & 0 & 0 & 0 & 0 & 0 & 0 \\
0 & 0 & 0 & 0 & 0 & 0 & 0 & 0 & 0 & 0 & 0 \\
0 & 0 & 0 & 0 & 0 & 0 & 0 & 0 & 0 & 0 & 0 \\
\hline
\end{tabular}

Table 2: Example of the best angle that determines the fit of the boundary of the iris

\begin{tabular}{lcccc}
\hline $\mathrm{XC}$ & YC & Angle value & Radius & Angle \\
\hline 289 & 276 & 97 & 10 & 45 \\
\hline
\end{tabular}

equivalent rectangular to transform the iris template from Cartesian to polar coordinates. Figure 11 shows an example of the normalized irises. After iris normalization stage there are two steps described as: 
(a)

(b)

(c)
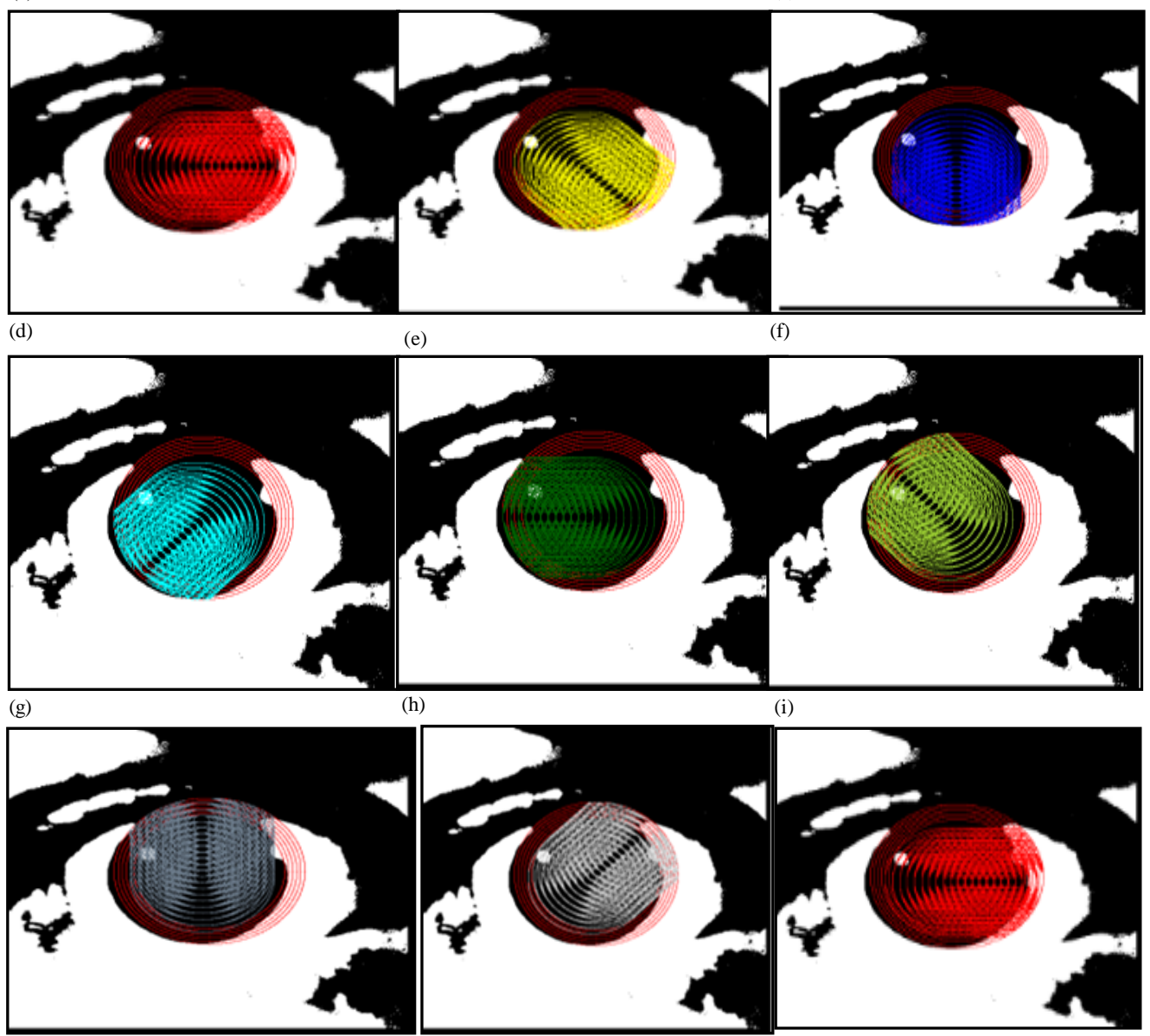

Fig. 9a-1): Circular distribution of angles
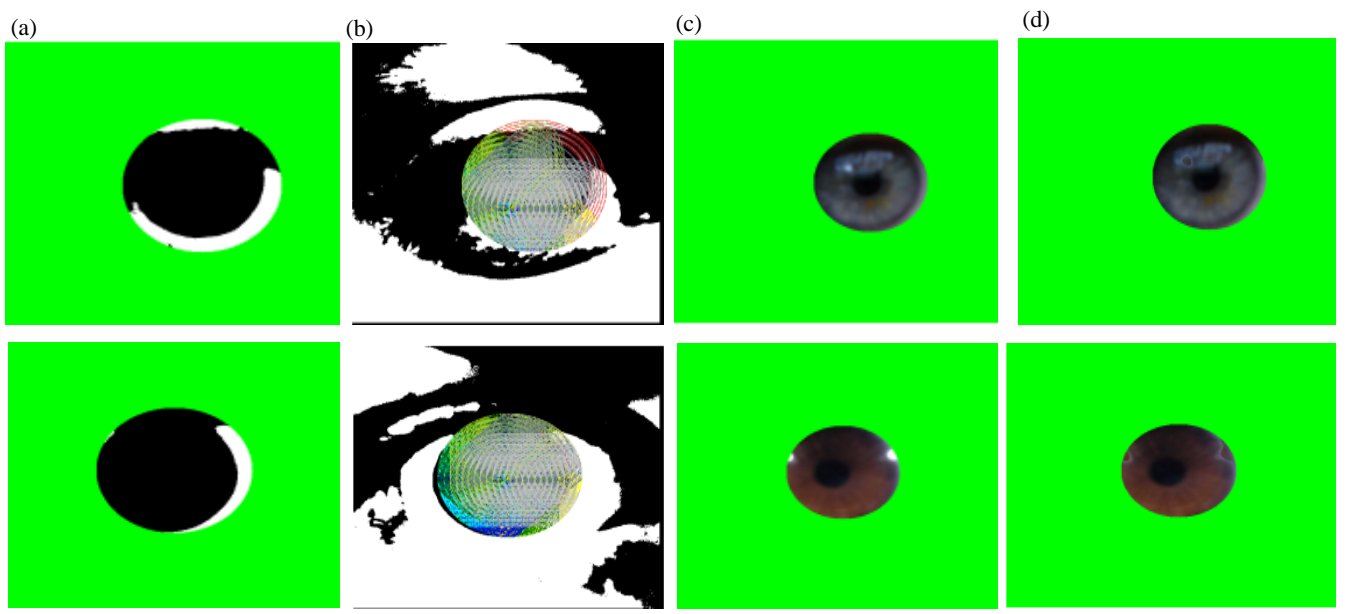

Fig. 10: Iris Segmentation output; a) Remove interpolation; b) Circular distribution of angles; c) Segmented iris and d) Remove interpolation after segmented iris

Step 1; iris enhancement with histogram equalization: Because most of the iris images have lowed contrasts and also may have non-uniform brightness dues to the changes in lights sources positions, the proposed 
enhancement method (histograme qualization) of normalized iris image which is very important before applying features extraction phase. Examples of iris enhancement results are presented in Fig. 12b.

Step 2; Canny edge detection filter CED: It is applied to find the weak edges of iris normalization that help us to provide the necessary information for extracting the iris features. Examples of CED results are presented in Fig. 12c.

Iris feature extraction stage: It aims to extract iris code from iris normalization image. This task is applied in both enrolment and recognition phases. In this study, the feature was extracted by using Zernike moments which is a complex set of radial orthogonal polynomials andmore grown orthogonal invariant moments. It can structure any higher moments with its rotation invariance. The extracted features have a small connection, little redundancy, strong noise immunity. The 9-order moments have been used to describe key frames feature which results in a feature vector of size $(9 \times 1)$ as shown in the Table 3 and Fig. 13.

(a)

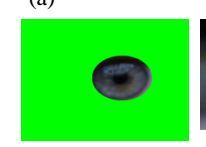

(b)
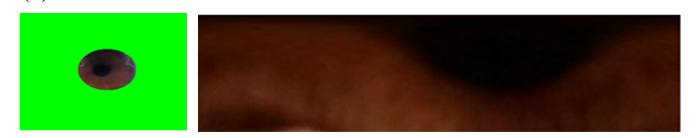

Fig. 11a, b): Example of normalization process output (a)

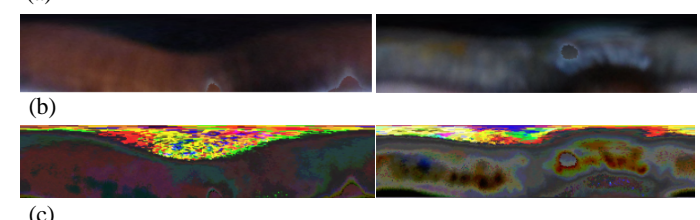

(c)

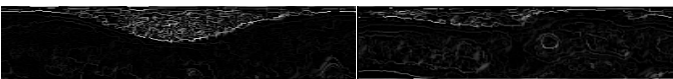

Fig. 12: Examples of iris enhancement; a) Iris normalization; b) Iris histogram equalization and c) Weak edge of canny edge detection
Pattern matching stage: The process of measuring the degree of similarity between two templates is referred to as matching. The measures used for the proposed system is the K-Nearest Neighbors (KNN). The KNN classifier is used to compare the feature vector for the test image and the iris images stored in the database.

MICHE dataset: The MICHE dataset-created by BIP Lab of the University of Salerno- contains iris acquired using three different mobile devices: iPhone 5S, Samsung Galaxy S4 and a Samsung Galaxy Tab II. The images in the database arecaptured by the subjects holding the capturing device by herself/himself (De Marsico et al., 2015). The subjects were asked to capture their own iris images using both front and rear camera of these devices in two different scenarios-indoor and outdoor. There is a minimum of 40 images per subject. The average capture distances for smartphones were $10 \mathrm{~cm}$ for the front camera and about $13 \mathrm{~cm}$ for the rear camera. For the Tab, this stand-off distance is about $5 \mathrm{~cm}$. The types of noise present in MLCHE-I dataset scenarios include:

Reflexes: Sources of artificial light, natural light sources, persons or objects on site during the acquisition, defocus blur. Motion blur: this is due to the involuntary movement of the eye, the head, the hand that holds the device.

Occlusions: Eyelids, eyeglasses, eyelashes, hair, shadows

Device: Artifacts due to the low resolution and/or to the specific noise of the device; Off-axis gaze.

Variable demonstration and different color dominants are present in this dataset. Examples of images in MICHE database are given in Fig. 14.

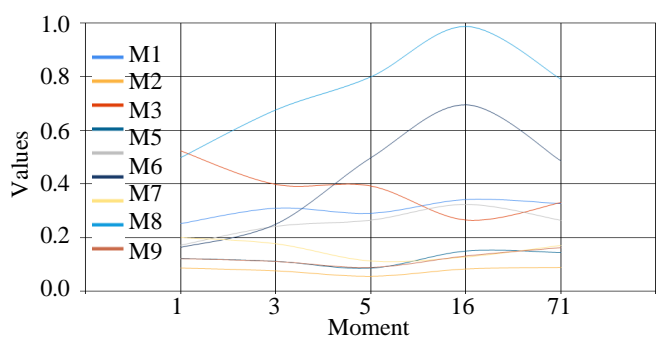

Fig. 13: Features extraction drawing result with Zernike moment

Table 3: Features extraction result with Zernike moments

\begin{tabular}{lcccccccccc}
\hline Class No. & M1 & M2 & M3 & M4 & M5 & M6 & M7 & M8 & M9 \\
\hline 1 & 0.252305 & 0.086455 & 0.523312 & 0.122212 & 0.171865 & 0.163795 & 0.200049 & 0.498598 & 0.121590 \\
3 & 0.309819 & 0.076150 & 0.398277 & 0.111649 & 0.242049 & 0.250276 & 0.177190 & 0.676177 & 0.111094 \\
5 & 0.290645 & 0.055843 & 0.392374 & 0.086213 & 0.265156 & 0.497733 & 0.112628 & 0.799429 & 0.088979 \\
16 & 0.342144 & 0.083034 & 0.265529 & 0.149961 & 0.323966 & 0.695330 & 0.127881 & 0.987421 & 0.131521 \\
71 & 0.326447 & 0.089172 & 0.330725 & 0.144146 & 0.264535 & 0.486137 & 0.170944 & 0.790766 & 0.162774 \\
\hline
\end{tabular}



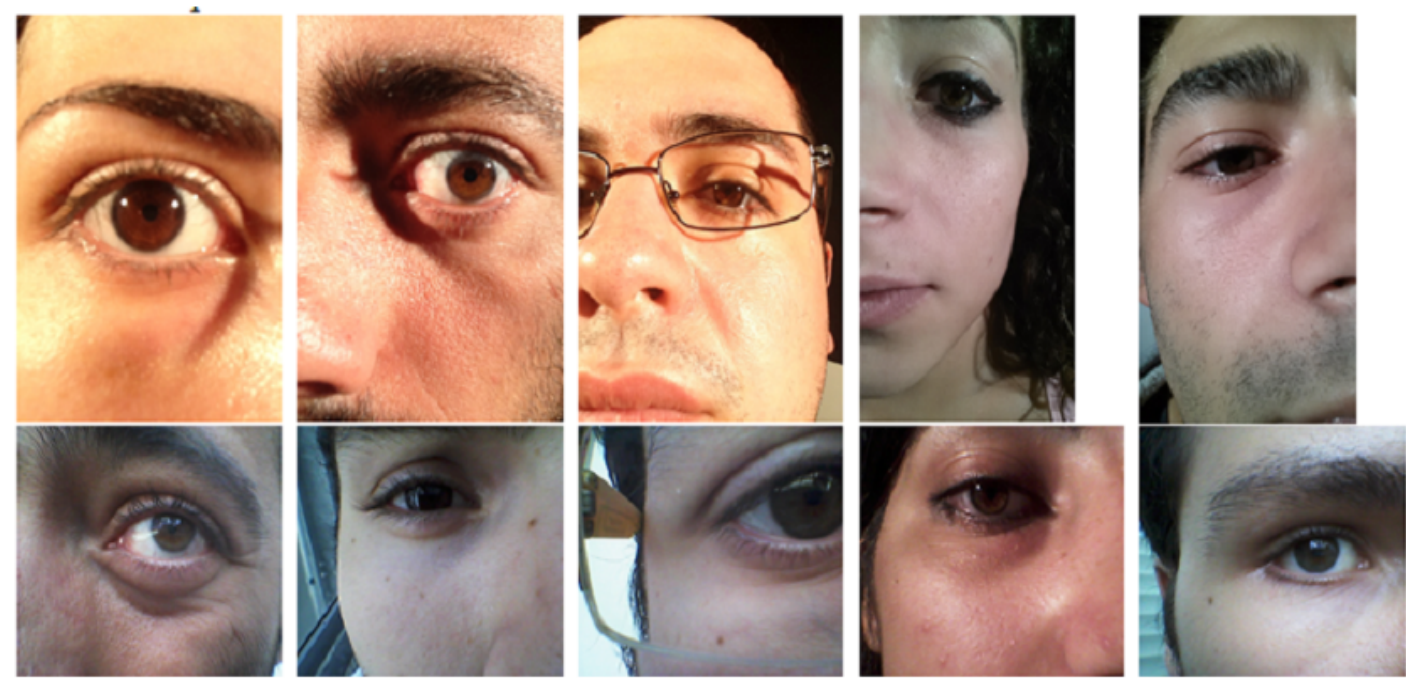

Fig. 14:Examples of images from the MICHE database: the first row contains images capturedusing the rear-facing camera and the second row contains images captured using a front-face camera
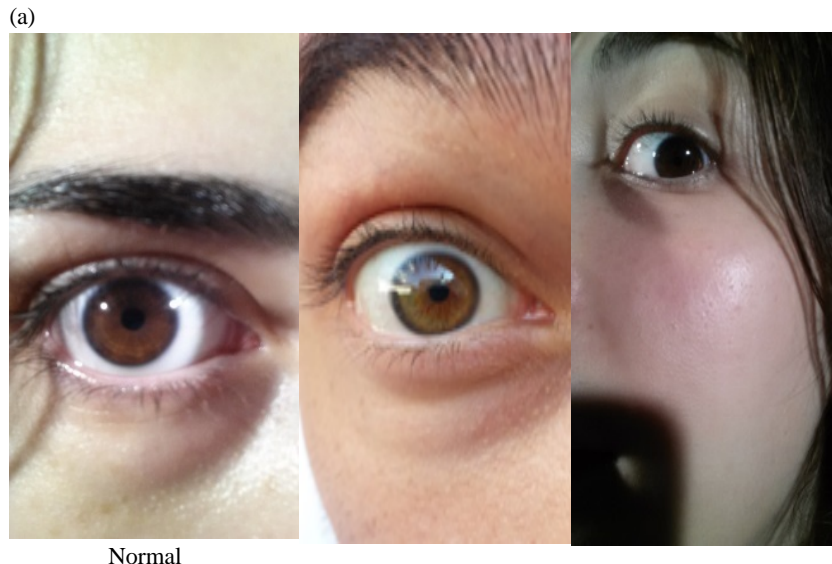

Reflection
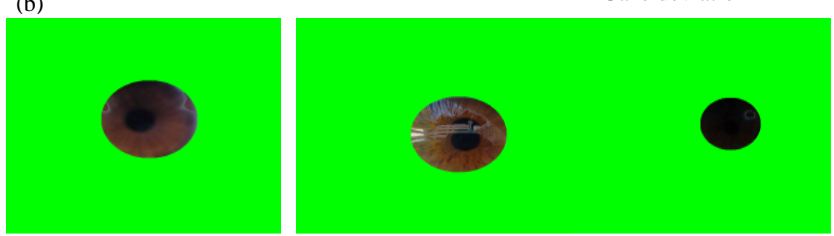

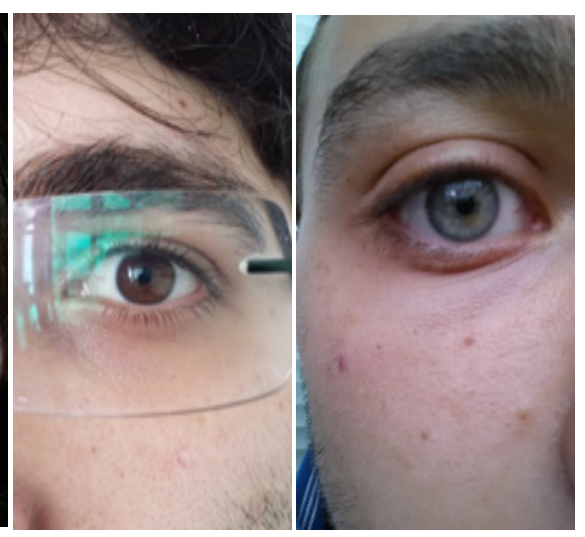

Eyeglasses

At a distance

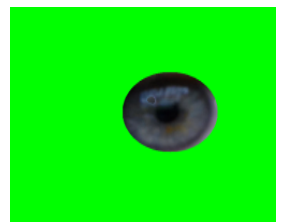

Fig. 15: Results of iris segmentation on Galaxy Samsung S4 dataset; a) Original images and b) Iris segmented

\section{RESULTS AND DISCUSSION}

The experiments have been conducted on the (MICHE-I dataset (SG4)), a database composed by 75 subjects with at least 16 images per subject, captured in different illumination conditions and different camera (front and rear) camera. In the experiment, we selected 4 photos acquired with the front camera and 4 photos acquired with the rear camera for the SG4 device, for a total of $(8$ picture per subject for training and 8 pictures per subject for testing) with image size $520 \times 520$ pixel. It must be noted that MICHE is a very challenging database, containing pictures affected by many noise factors. Iris recognition system performances could be improved by pre-processing iris images to remove the noise. It can be also seen that the proposed system proves its capability in segmenting irises affected by reflections, eye glass obstruction, gaze deviation and eyelid occlusion as demonstrated in Fig. 15. 
Table 4: The results of average FAR, FRR and accuracy of the proposed system for MICHE-I

\begin{tabular}{lllccc}
\hline Databases & Feature extraction methods & Matching & FRR (\%) & FAR (\%) & Accuracy (\%) \\
\hline MICHE-I GS4 & ZMs & KNN & 0.21 & 0.17 & 80 \\
\hline
\end{tabular}

Table 5: The testing performance of the proposed system

Zernike moments ( KNN)

\begin{tabular}{|c|c|c|c|c|c|c|c|c|c|c|c|}
\hline \multicolumn{3}{|c|}{---------Indoor-front camera--------- } & \multicolumn{3}{|c|}{----------Indoor-rear camera-------- } & \multicolumn{3}{|c|}{--------Outdoor-front camera------ } & \multicolumn{3}{|c|}{--------Outdoor-rear c amera--------- } \\
\hline $\mathrm{TP}=100$ & $\mathrm{FP}=15$ & 115 & $\mathrm{TP}=103$ & $\mathrm{FP}=13$ & 116 & $\mathrm{TP}=96$ & $\mathrm{FP}=19$ & 115 & $\mathrm{TP}=25$ & $\mathrm{FP}=21$ & 116 \\
\hline $\mathrm{FN}=22$ & $\mathrm{TN}=89$ & 111 & $\mathrm{FN}=25$ & $\mathrm{TN}=27$ & 112 & $\mathrm{FN}=31$ & $\mathrm{TN}=79$ & 110 & $\mathrm{FN}=33$ & $\mathrm{TN}=73$ & 106 \\
\hline 122 & 104 & 226 & 128 & 100 & 228 & 127 & 98 & 225 & 128 & 94 & 222 \\
\hline
\end{tabular}

The proposed system has been implemented using a laptop computer (processor:An Intel (R) Core (TM) i3 CPUM380@2.53 GHzwith 32-bit Operating System and 4GB RAM), the operating system is Windows 7. The programming language Visual $\mathrm{CH}$ is used to build and developed the required software.

The results of FAR, FRR and accuracy in proposed iris recognition in smartphones are presented in Table 4 for MICHE-I (GS4) database samples using the features belong for the approach (Zernike moments). Table 5 presents the testing performance of the proposed system for MICHE-I GS4.The proposed system has an $80 \%$ average accuracy by using Zernike moments in feature extraction and KNN for matching compared with others systems (Table 4).

\section{CONCLUSION}

In this study, a new system of predicate iris center is presented based on the circular histogram with challenging database conditions in addition, an adaptive segmentation algorithm for iris images acquired in unconstrained environments has been proposed. This method improves the unconstrained iris segmentation process in two ways; Firstly, it predicates iris boundaries through predicate center and radius of iris; Secondly, itdetects actual iris boundary based onthe circular distribution of angles method to find the actual radius and center for each iris. Our experiments have shown that circular distribution of angles was very robust in finding the boundary of iris even in noisy and difficult conditions in smartphones environments. An efficient algorithm for iris recognition using Zernike moment in feature extraction has been developed. Also, we have used classification KNN method. From results, it is concluded that KNN classification is more reliable for obtaining accurate results in smartphone environments. The proposed method yields (80\%) average accuracy rate. Even though all mobile devices are equipped with various biometric sensors such as cameras, microphones and fingerprint sensors, more research is needed in order to robustly consolidate the different biometric modalities on a single mobile device. Multimodal biometrics promises to be the future of mobile authentication more secure.

\section{REFERENCES}

Barra, S., A. Casanova, F. Narducci and S. Ricciardi, 2015. Ubiquitous iris recognition by means of mobile devices. Pattern Recognit. Lett., 57: 66-73.

De Marsico, M., M. Nappi, D. Riccio and H. Wechsler, 2015. Mobile Iris Challenge Evaluation (MICHE)-I, biometric iris dataset and protocols. Pattern Recognit. Lett., 57: 17-23.

Galdi, C. and J.L. Dugelay, 2017. FIRE: Fast iris recognition on mobile phones by combining colour and texture features. Pattern Recognit. Lett., 91: 44-51.

Jillela, R.R. and A. Ross, 2015. Segmenting iris images in the visible spectrum with applications in mobile biometrics. Pattern Recognit. Lett., 57: 4-16.

Kim, D., Y. Jung, K.A. Toh, B. Son and J. Kim, 2016. An empirical study on iris recognition in a mobile phone. Exp. Syst. Appl., 54: 328-339.

Mitra, S. and M. Gofman, 2016. Biometrics in a Data Driven World: Trends, Technologies and Challenges. CRC Press, Boca Raton, Florida, USA., ISBN:9781315317069, Pages: 402.

Prabhakar, S., S. Pankanti and A.K. Jain, 2003. Biometric recognition: Security and privacy concerns. IEEE Secur. Priv., 1: 33-42.

Raja, K.B., R. Raghavendra, S. Venkatesh and C. Busch, 2017. Multi-patch deep sparse histograms for iris recognition in visible spectrum using collaborative subspace for robust verification. Pattern Recognit. Lett., 91: 27-36.

Raja, K.B., R. Raghavendra, V.K. Vemuri and C. Busch, 2015. Smartphone based visible iris recognition using deep sparse filtering. Pattern Recognit. Lett., 57: 33-42.

Thavalengal, S., 2016. Contributions to practical iris biometrics on smartphones. Ph.D Thesis, College of Engineering and Informatics, National University of Ireland, Galway, Ireland. 